\title{
ON DENSITY OF SMOOTH FUNCTIONS IN WEIGHTED SOBOLEV SPACES WITH VARIABLE EXPONENTS
}

\author{
V. V. ZHIKOV AND M. D. SURNACHEV
}

Dedicated to Nina Ural'tseva on the occasion of her anniversary.

\begin{abstract}
A sufficient condition for the density of smooth functions in the weighted Sobolev space with variable exponent is obtained. This condition is formulated in terms of the asymptotic behavior of the integrals of negative and positive powers of the weight.
\end{abstract}

\section{§1. INTRODUCTION}

In a bounded Lipschitz domain $\Omega \subset \mathbb{R}^{n}, n \geq 2$, a weight $\rho$ is given, which is a nonnegative measurable function integrable on $\Omega$. Also, we are given an exponent $p=p(x)$ measurable on $\Omega$ and satisfying the condition

$$
1<\alpha<p(x)<\beta<\infty .
$$

The norm, usually called a Luxemburg norm, is introduced in a standard way:

$$
\|f\|_{p(\cdot), \rho}=\inf \left\{\lambda>0: \int_{\Omega}\left|\lambda^{-1} f\right|^{p(x)} \rho \mathrm{d} x \leq 1\right\} .
$$

It should be mentioned that there are grounds to call it more appropriately a Kolmogorov-Minkowski norm. The class $L^{p(\cdot)}(\Omega ; \rho \mathrm{d} x)$ with this norm is a reflexive separable Banach space. If $\rho \equiv 1$, then we use the standard notation $\|\cdot\|_{p(\cdot)}$ for the Luxemburg norm in $L^{p(\cdot)}(\Omega)$.

We introduce the weighted Sobolev-Orlicz space

$$
W=\left\{u \in W_{0}^{1,1}(\Omega): \int_{\Omega}|\nabla u|^{p(x)} \rho \mathrm{d} x<\infty\right\}, \quad\|u\|_{W}:=\|\nabla u\|_{p(\cdot), \rho},
$$

assuming that

$$
\rho^{-1 / p} \in L^{p^{\prime}}(\Omega), \text { where } p^{\prime}(x)=\frac{p(x)}{p(x)-1} .
$$

Then, applying the generalized Hölder inequality

$$
\int_{\Omega}|\nabla u| \mathrm{d} x \leq 2\|\nabla u\|_{L^{p}(\Omega, \rho \mathrm{d} x)}\left\|\rho^{-1 / p}\right\|_{L^{p^{\prime}}(\Omega)}
$$

and recalling that $W_{0}^{1,1}(\Omega)$ is complete, it is easy to deduce the completeness of $W$. We denote by $H$ the closure of $C_{0}^{\infty}(\Omega)$ in the norm $\|\cdot\|_{W}$.

2010 Mathematics Subject Classification. Primary 46E35.

Key words and phrases. Density of smooth functions, Lavrentiev phenomenon, Sobolev-Orlicz spaces, variable exponent, Muckenhoupt classes.

Supported by RFBR (projects nos. 14-01-31341 and 14-01-00192). The results of $\S 4$ were obtained with the support of Russian Science Foundation (project no. 14-11-00398). 
Definition. We say that the weight $\rho$ is regular if $H=W$, i.e., when the smooth functions are dense in the Sobolev space.

The inequality $H \neq W$ expresses the so-called Lavrentiev phenomenon (see [1, 2, 3, 4]). Accordingly, if $H=W$, then the Lavrentiev phenomenon is absent.

When we need to consider Sobolev spaces with different exponents and weights, we shall write $W(\Omega, p(\cdot), \rho)$ and $H(\Omega, p(\cdot), \rho)$. Thus, with this notation, $W_{0}^{1,2}(\Omega)=$ $W(\Omega, 2,1)$ and $H_{0}^{1,2}(\Omega)=H(\Omega, 2,1)$.

In the case of a constant exponent $p=$ const the following facts are classical. For $\rho \equiv 1$, the fact that $H^{1, p}(\Omega)=W^{1, p}(\Omega)$, i.e., the density of $C^{\infty}(\Omega) \cap W^{1, p}(\Omega)$ in the classical Sobolev space $W^{1, p}(\Omega)$, with no assumptions about the smoothness of the domain, was proved in the well-known paper [5] by Meyers and Serrin. If a certain smoothness of the boundary is assumed, then the density of $C^{\infty}(\bar{\Omega})$ in $W^{1, p}(\Omega)$ can also be proved. For a weight that is not separated uniformly away from zero and infinity, there is a rather general condition ensuring that $H^{1, p}(\Omega, \rho \mathrm{d} x)=W^{1, p}(\Omega, \rho \mathrm{d} x)$ (i.e., that the functions of class $C^{\infty}(\Omega)$ are dense in the latter space) - the weight $\rho$ should belong to the Muckenhoupt class $A_{p}$. In this case the proof repeats that of Meyers-Serrin word for word, because there an estimate of the maximal operator is available, which implies the boundedness of the classical smoothing operators. These matters are accounted in detail in 6.

We note that the methods based on smoothing integral operators do not allow one to go beyond the scope Muckenhoupt weights. A classical result (see, e.g., 7]) states, that the uniform boundedness of the class of smoothing integral operators in $L^{p}(\Omega, \omega \mathrm{d} x)$ is equivalent to the fact that $\omega \in A_{p}$. Thus, other techniques are required.

In the recent paper [8], Zhikov uses Lipschitz truncations method to prove the following theorem.

Theorem 1. Suppose that $p=2$, the weight $\rho$ has the form $\rho=\omega \omega_{0}$ with $\omega_{0} \in A_{2}$, and

$$
\liminf _{t \rightarrow \infty} \frac{\left(\int_{\Omega} \omega^{t} \omega_{0} \mathrm{~d} x\right)^{1 / t} \cdot\left(\int_{\Omega} \omega^{-t} \omega_{0} \mathrm{~d} x\right)^{1 / t}}{t^{2}}<\infty .
$$

Then the weight $\rho$ is regular.

In the general case it is not easy to verify the condition of Theorem 1. However, the following sufficient condition known as the De Giorgi conjecture can easily be obtained.

Theorem 2. Suppose that $p=2$, the weight $\rho$ has the form $\rho=\omega \omega_{0}$ with $\omega_{0} \in A_{2}$, and

$$
\exp (t \omega), \exp \left(t \omega^{-1}\right) \in L^{1}\left(\Omega, \omega_{0} \mathrm{~d} x\right)
$$

for a certain $t>0$. Then the weight $\rho$ is regular.

Proof. Since $\exp (t \omega) \in L^{1}\left(\Omega, \omega_{0} \mathrm{~d} x\right)$, expanding the exponent in a series and using Cauchy's convergence test, we obtain

$$
\limsup _{n \rightarrow \infty}\left(\int_{\Omega} \frac{t^{n} \omega^{n}}{n !} \omega_{0} \mathrm{~d} x\right)^{1 / n} \leq 1
$$

Then the Stirling formula yields

$$
\limsup _{n \rightarrow \infty} \frac{1}{n}\left(\int_{\Omega} \omega^{n} \omega_{0} \mathrm{~d} x\right)^{1 / n} \leq \frac{1}{e t} .
$$

Similarly, since $\exp \left(t \omega^{-1}\right) \in L^{1}\left(\Omega, \omega_{0} \mathrm{~d} x\right)$, we obtain

$$
\limsup _{n \rightarrow \infty} \frac{1}{n}\left(\int_{\Omega} \omega^{-n} \omega_{0} \mathrm{~d} x\right)^{1 / n} \leq \frac{1}{e t} .
$$


Thus, the condition of Theorem 1 is satisfied.

In a similar way, it is possible to obtain similar theorems for any fixed $p>1$.

Theorem 3. Suppose that $p=$ const $>1$, the weight $\rho$ has the form $\rho=\omega \omega_{0}$ with $\omega_{0} \in A_{p}$, and

$$
\liminf _{t \rightarrow \infty} \frac{\left(\int_{\Omega} \omega^{t} \omega_{0} \mathrm{~d} x\right)^{1 / t} \cdot\left(\int_{\Omega} \omega^{-t} \omega_{0} \mathrm{~d} x\right)^{1 / t}}{t^{p}}<\infty .
$$

Then the weight $\rho$ is regular.

Note that the class of admissible weights in Theorem 3 grows as $p$ increases. In this paper we generalize this result to the case of a variable exponent $p=p(x)$. Previously, a short communication [9] was published on this subject.

We recall some facts concerning the density of smooth functions in Sobolev spaces with variable exponent $p=p(x)$. Even without a weight function, these results are nontrivial, because in general the continuity of the shift operator may fail, see [10. In connection with the density problem for smooth functions, Zhikov [2, 3] and Fan [11] (see also [12]) introduced the well-known logarithmic condition

$$
|p(x)-p(y)| \leq \frac{k_{0}}{\ln \frac{1}{|x-y|}}, \quad|x-y|<1,
$$

and showed that it ensures the uniform boundedness of the classical smoothing operators

$$
T_{\varepsilon} f=f * \varphi_{\varepsilon}=\int f(y) \varphi_{\varepsilon}(x-y) \mathrm{d} y
$$

in $L^{p(\cdot)}$. Thus, $C_{0}^{\infty}\left(\mathbb{R}^{n}\right)$ is dense in $W^{1, p(\cdot)}\left(\mathbb{R}^{n}\right)$. For a bounded Lipschitz domain, the same conditions on the exponent $p(\cdot)$ (logarithmic modulus of continuity) guarantees that $C_{0}^{\infty}(\Omega)$ is dense in $W_{0}^{1, p(\cdot)}(\Omega)$ and $C^{\infty}(\bar{\Omega})$ is dense in $W^{1, p(\cdot)}(\Omega)$. Similar results were also proved by other authors, e.g., by S. G. Samko 13 and L. Diening [14. It has also been discovered that the logarithmic condition (1.4) is optimal for facts like the boundedness of the maximal operator in the Lebesgue space $L^{p(\cdot)}\left(\mathbb{R}^{n}\right)$ with variable exponent (see [15, 16, 17, 18, 19]), the boundedness of some classical operators [20], and many others.

V. V. Zhikov presented examples showing that the replacement of the logarithmic modulus of continuity (1.4) with $k \ln ^{-s} \frac{1}{|x-y|}$, where $0<s<1$, leads to the loss of the density of smooth functions in Sobolev space.

First, we state a partial version of the main result of this paper.

Theorem 4. Suppose that the exponent $p(\cdot)$ satisfies (1.1) and (1.4) and that the following condition is fulfilled:

$$
\liminf _{t \rightarrow \infty}\left(\int_{\Omega} \rho^{-t} \mathrm{~d} x\right)^{1 / t}\left(\int_{\Omega}\left(t^{-p(x)} \rho\right)^{t} \mathrm{~d} x\right)^{1 / t}<\infty .
$$

Then the weight $\rho$ is regular.

To formulate the result in full generality, we need the definition of the Muckenhoupt classes with variable exponents, which we give later.

It is known (see [21]) that the smooth functions are dense in $W^{1, p(\cdot)}\left(\mathbb{R}^{n}\right)$ under a weaker condition on the continuity modulus $r(t)$ of $p(\cdot)$, compared to the logarithmic condition (1.4), namely, it suffices to assume the divergence of the integral $\int_{0}^{1} t^{-1+\frac{r(t) n}{\alpha}} \mathrm{d} t=$ $\infty$. In particular, this condition is satisfied for the continuity modulus $r(t)=\frac{k \ln \ln \frac{1}{t}}{\ln \frac{1}{t}}$ for $0<k \leq \alpha / n$. Thus, the following question arises: does Theorem 4 admit generalization 
to the case of an exponent with continuity modulus weaker than logarithmic? We have not managed to accomplish that yet.

Having at hand the density of smooth functions with compact support in the Sobolev space with zero boundary condition, we can, under the assumptions of our theorems, recapture the result of Meyers-Serrin, i.e., prove the density of smooth functions in the Sobolev space without boundary conditions. We assume that the norm in that space is given by the expression

$$
\|u\|_{p(\cdot), \rho}+\|\nabla u\|_{p(\cdot), \rho} \cdot
$$

Accordingly, we need to show that every function belonging to

$$
W^{1, p(\cdot)}(\Omega ; \rho \mathrm{d} x):=\left\{u \in W^{1,1}(\Omega):\|u\|_{p(\cdot), \rho}+\|\nabla u\|_{p(\cdot), \rho}<\infty\right\}
$$

can be approximated by functions of class $C^{\infty}(\Omega)$. Consider the partition of the domain into the layers

$$
\Omega_{j}=\left\{x \in \Omega: j^{-1}<\operatorname{dist}(x, \partial \Omega)<(j-1)^{-1}\right\}, \quad j \geq 1,
$$

where for $j=1$ we put $(j-1)^{-1}=+\infty$. Denote

$$
D_{1}=\bar{\Omega}_{1} \cup \Omega_{2}, \quad D_{j}=\Omega_{j-1} \cup \bar{\Omega}_{j} \cup \Omega_{j+1}, \quad j \geq 2 .
$$

We select a partition of unity: $\xi_{j} \geq 0, \sum_{j=1}^{\infty} \xi_{j}=1, \xi_{j}=1$ on $\Omega_{j}, \xi_{j} \in C_{0}^{\infty}\left(D_{j}\right)$. Denote $u_{j}=u \xi_{j}$. In each of the domains $D_{j}$, the weight satisfies condition (5.11) of the main theorem. Thus, there exists a function $v_{j} \in C_{0}^{\infty}\left(D_{j}\right)$ such that $\left\|v_{j}-u_{j}\right\|_{W\left(D_{j}\right)}<\varepsilon / 2^{j}$. Next, we have

$$
\left\|u-\sum v_{j}\right\|_{W(\Omega)} \leq \sum\left\|u_{j}-v_{j}\right\|_{W\left(D_{j}\right)}<\varepsilon .
$$

Thus, the initial function is approximated by smooth functions in the norm of $W$.

We show that in each domain $D_{j}$ the functions $v \in C_{0}^{\infty}\left(D_{j}\right)$ satisfy the inequality

$$
\|v\|_{p(\cdot), \rho} \leq C_{j}\|\nabla v\|_{p(\cdot), \rho} .
$$

We recall that by the embedding inequality $[22,23$, we have

$$
\|v\|_{\frac{n p(\cdot)}{n-1}} \leq c_{j}\|\nabla v\|_{p(\cdot)} .
$$

Now, we select two numbers $\kappa>1$ and $\gamma \in\left(\frac{1}{\alpha}, 1\right)$ such that $\kappa \leq \frac{n \gamma}{n-1}$. Using the Hölder inequality and (1.6), we obtain

$$
\begin{aligned}
\|v\|_{p(\cdot), \rho} & =\left\|v \rho^{1 / p(\cdot)}\right\|_{p(\cdot)} \leq 2\|v\|_{\kappa p(\cdot)} \cdot\left\|\rho^{1 / p}\right\|_{\frac{\kappa p(\cdot)}{\kappa-1}} \\
& \leq 2 c_{j}\|\nabla v\|_{\kappa_{1} p(\cdot)} \cdot\left\|\rho^{1 / p}\right\|_{\frac{\kappa p(\cdot)}{\kappa-1}} \\
& =2 c_{j}\left\|\rho^{-1 / p(\cdot)} \rho^{1 / p(\cdot)} \nabla v\right\|_{\gamma p(\cdot)} \cdot\left\|\rho^{1 / p}\right\|_{\frac{\kappa p(\cdot)}{\kappa-1}} \\
& \leq 4 c_{j}\|\nabla v\|_{p(\cdot), \rho} \cdot\left\|\rho^{-1 / p}\right\|_{\frac{\gamma p}{1-\gamma}} \cdot\left\|\rho^{1 / p}\right\|_{\frac{\kappa p(\cdot)}{\kappa-1}} .
\end{aligned}
$$

Thus, the initial function is approximated in the norm (1.5).

The modern state of the theory of Sobolev spaces with variable exponents is covered in detail in the survey [24] and the book [22].

\section{$\S 2$. EXAmples}

Here we analyze some examples of the presence/absence of the density of smooth functions in Sobolev spaces. In our situation, two circumstances must be taken into account: the presence of the weight $\rho$ and the variable exponent $p(\cdot)$. By $B_{R}$ we denote the ball of radius $R$ centered at the origin.

We begin with known examples of the presence/absence of the density of smooth functions for a variable exponent. The classical example where $H \neq W$ in the case of a 
discontinuous exponent was constructed by Zhikov in the paper [1] dated back to 1986 . In that example, the exponent is defined on $B=\{|x| \leq 1 / 2\} \subset \mathbb{R}^{2}$ by the expression

$$
p(x)=\left\{\begin{array}{ll}
\alpha & \text { if } x_{1} x_{2}>0, \\
\beta & \text { if } x_{1} x_{2}<0,
\end{array} \quad 1<\alpha<2<\beta<\infty .\right.
$$

This example was inspired by considering the composite materials with "chessboard" structure. At the same time, in a simpler case of a piecewise constant exponent, defined in the same ball $B$ by

$$
p(x)=\left\{\begin{array}{ll}
\alpha & \text { if } x_{2}>0, \\
\beta & \text { if } x_{2}<0,
\end{array} \quad 1<\alpha<\beta<\infty,\right.
$$

the density of smooth functions in the corresponding Sobolev space with exponent $p(\cdot)$ holds true, i.e., $H=W$. Indeed, consider the even extension $v\left(x_{1}, x_{2}\right)=u\left(x_{1},-x_{2}\right)$, $x_{2}>0$, a given function $u \in W$ from the subdomain with the greater exponent, $B_{-}=$ $B \cap\left\{x_{2}<0\right\}$, to the subdomain $B_{+}=B \cap\left\{x_{2}>0\right\}$ with the lesser value of the exponent. Consider the difference $z=u-v \in W_{0}^{1, \alpha}\left(B_{+}\right)$. There exists a sequence of $z_{\varepsilon} \in C_{0}^{\infty}\left(B_{+}\right)$ approximating $z$ in the norm of $W_{0}^{1, \alpha}\left(B_{+}\right)$. Put

$$
u_{\varepsilon}=\left\{\begin{array}{lll}
u & \text { on } & B_{-}, \\
v+z_{\varepsilon} & \text { on } & B_{+} .
\end{array}\right.
$$

The functions $u_{\varepsilon} \in W_{0}^{1, \beta}(B)$ approximate $u$ in the norm of $W$. In their turn, the functions $u_{\varepsilon}$ admit approximation by smooth functions in the norm of $W_{0}^{1, \beta}(B)$, and thus, in the norm of $W$.

In the paper [21], Zhikov produced a rather general method of constructing examples where $H \neq W$ : for four nonoverlapping sectors of a disk $A_{(1)-(4)}$ arranged counterclockwise, the simultaneous convergence of the integrals

$$
\int_{A_{(1)} \cup A_{(3)}}|x|^{-p^{\prime}(x)} \mathrm{d} x<\infty, \quad \int_{A_{(2)} \cup A_{(4)}}|x|^{-p(x)} \mathrm{d} x<\infty
$$

guaranties the absence of the density of smooth functions. We recall how this example is constructed. Let $\Gamma_{j}$ denote the unit circle arcs corresponding to the sectors $A_{(j)}$. Let $u \in C_{0}^{\infty}\left(B_{1}\right)$. Using the Newton-Leibniz formula, we obtain

$$
u(\theta, R)=u(0)+\int_{0}^{R} \frac{\partial u}{\partial r}(\theta, r) \mathrm{d} r=-\int_{R}^{1} \frac{\partial u}{\partial r}(\theta, r) \mathrm{d} r .
$$

Integrating over the angles of the first and the third $\operatorname{arcs} \Gamma_{1}$ and $\Gamma_{3}$, we see that

$$
\begin{aligned}
& \frac{1}{\left|\Gamma_{i}\right|} \int_{\Gamma_{i}}|u(\theta, R)| \mathrm{d} \theta \leq \frac{1}{\left|\Gamma_{i}\right|} \int_{A_{(i)}} r^{-1}|\nabla u| \mathrm{d} x \\
& \quad \leq \frac{1}{\left|\Gamma_{i}\right|} \int_{A_{(i)}}|x|^{-p^{\prime}(x)} \mathrm{d} x+\frac{1}{\left|\Gamma_{i}\right|} \int_{A_{(i)}}|\nabla u|^{p(x)} \mathrm{d} x, \quad i=1,3,
\end{aligned}
$$

and also

$$
\begin{aligned}
& \left|\frac{1}{\left|\Gamma_{i}\right|} \int_{\Gamma_{i}}(u(\theta, R)-u(0)) \mathrm{d} \theta\right| \leq \frac{1}{\left|\Gamma_{i}\right|} \int_{A_{(i)} \cap B_{R}} r^{-1}|\nabla u| \mathrm{d} x \\
& \quad \leq \frac{1}{\left|\Gamma_{i}\right|} \int_{A_{(i)} \cap B_{R}}|x|^{-p^{\prime}(x)} \mathrm{d} x+\frac{1}{\left|\Gamma_{i}\right|} \int_{A_{(i)} \cap B_{R}}|\nabla u|^{p(x)} \mathrm{d} x, \quad i=1,3 .
\end{aligned}
$$


Hence,

$$
\begin{aligned}
& \left|\frac{1}{\left|\Gamma_{1}\right|} \int_{\Gamma_{1}} u(\theta, R) \mathrm{d} \theta-\frac{1}{\left|\Gamma_{3}\right|} \int_{\Gamma_{3}} u(\theta, R) \mathrm{d} \theta\right| \\
& \quad \leq\left(\left|\Gamma_{1}\right|^{-1}+\left|\Gamma_{3}\right|^{-1}\right)\left(\int_{\left(A_{(1)} \cup A_{(3)}\right) \cap B_{R}}|x|^{-p^{\prime}(x)} \mathrm{d} x+\int_{\left(A_{(1)} \cup A_{(3)}\right) \cap B_{R}}|\nabla u|^{p(x)} \mathrm{d} x\right) .
\end{aligned}
$$

This estimate is also valid for $u \in H$. Thus,

$$
\lim _{R \rightarrow 0}\left(\frac{1}{\left|\Gamma_{1}\right|} \int_{\Gamma_{1}} u(\theta, R) \mathrm{d} \theta-\frac{1}{\left|\Gamma_{3}\right|} \int_{\Gamma_{3}} u(\theta, R) \mathrm{d} \theta\right)=0, \quad u \in H .
$$

Now, we construct a function belonging to the Sobolev space $W$ and such that this identity fails. Consider the function $u_{0}=\left(1-r^{2}\right) \Phi(\theta)$, where $\Phi(\cdot)$ is a $2 \pi$-periodic function such that

$$
0 \leq \Phi \leq 1,\left.\quad \Phi\right|_{\Gamma_{1}}=0,\left.\quad \Phi\right|_{\Gamma_{3}}=1, \quad \operatorname{supp} \Phi^{\prime} \subset \Gamma_{2} \cup \Gamma_{4} .
$$

For such a function $u_{0}$, the limits in (2.3) obviously differ. On the other hand, it is easily seen that $\left|\nabla u_{0}\right| \leq C\left(r+r^{-1}\right)$. Thus, $u_{0} \in W$ :

$$
\int_{B}\left|\nabla u_{0}\right|^{p(x)} \mathrm{d} x<\infty
$$

For example, in order to prove that $H \neq W$ for an exponent as in (2.1), it suffices to consider the sectors of the disk corresponding to the $\operatorname{arcs} \Gamma_{j}=\{\pi(j-1) / 2 \leq \theta<\pi j / 2\}$, and set

$$
\Phi(\theta)= \begin{cases}\sin \theta, & 0<\theta<\frac{\pi}{2}, \\ 1, & \frac{\pi}{2}<\theta<\pi, \\ -\cos \theta, & \pi<\theta<\frac{3 \pi}{2}, \\ 0, & \frac{3 \pi}{2}<\theta<2 \pi .\end{cases}
$$

In the sectors $A_{(1)}$ and $A_{(3)}$ we have $p^{\prime}(x)=\beta^{\prime}<2$, and in the sectors $A_{(2)}$ and $A_{(4)}$ we have $p(x)=\alpha<2$, so that the integrals in (2.2) converge. Note that for the exponent given in (2.1), the codimension of $H$ in $W$ equals one (see, e.g., 24]).

Next we present an interesting example illustrating this result. In the ball $B=\{|x|<$ $\left.r_{0}\right\} \subset \mathbb{R}^{2}$ with a sufficiently small radius $r_{0}<e^{-2}$, consider the exponent

$$
p(x)=2+k \chi(r) \cos 2 \theta, \quad \chi(r)=\frac{\ln \ln \frac{1}{r}}{\ln \frac{1}{r}}, \quad k=\text { const } .
$$

We show that $H \neq W$ for $k>1$. Let $A_{(1)-(4)}$ denote the sectors

$$
|\theta|<\delta, \quad\left|\theta-\frac{\pi}{2}\right|<\delta, \quad|\theta-\pi|<\delta, \quad\left|\theta-\frac{3 \pi}{2}\right|<\delta
$$

with a sufficiently small opening $2 \delta$, namely, we assume that

$$
p(x) \leq 2-k_{1} \chi(r), \quad x \in A_{(2)} \cup A_{(4)}, \quad p^{\prime}(x) \leq 2-k_{1} \chi(r) \quad x \in A_{(1)} \cup A_{(3)}
$$

for some value of $k_{1} \in(1, k)$. Then conditions (2.2) are satisfied. Indeed,

$$
\int_{A_{(1)} \cup A_{(3)}}|x|^{-p^{\prime}(x)} \mathrm{d} x \leq \int_{0}^{1 / 2} r^{k_{1} \chi(r)-1} \mathrm{~d} r=\int_{0}^{1 / 2} \frac{d r}{r|\ln r|^{k_{1}}}<\infty,
$$

and the same for $A_{(2)} \cup A_{(4)}$. Calculations show that the continuity modulus of the exponent (2.4) in the disk of radius $1 / 30$ is no greater than $5 k \chi(\cdot)$.

The construction specified above fits for producing counterexamples even when a weight function is present. We state it in the following way. 
Theorem. If for four consecutively arranged nonoverlapping sectors $A_{1}-A_{4}$ of a disk $B_{R} \subset \mathbb{R}^{2}$ we have

$$
\int_{A_{(1)} \cup A_{(3)}}|x|^{-p^{\prime}(x)} \rho^{1 /(1-p(x))} \mathrm{d} x<\infty, \quad \int_{A_{(2)} \cup A_{(4)}}|x|^{-p(x)} \rho \mathrm{d} x<\infty,
$$

then $H\left(B_{R}, p(\cdot), \rho\right) \neq W\left(B_{R}, p(\cdot), \rho\right)$.

For $p=2$, we get the following example, given by Zhikov in [6]. On the disk $\{|x|<$ 1\} $\subset \mathbb{R}^{2}$ consider the weight

$$
\rho(x)= \begin{cases}(a(r))^{-1} & \text { if } \quad x_{1} x_{2}>0 \\ a(r) & \text { if } \quad x_{1} x_{2}<0\end{cases}
$$

where the function $a(\cdot)$ is continuous and strictly positive on any interval $(\varepsilon, 1), \varepsilon>0$,

$$
a(r) \geq c(\varepsilon)>0, \quad r>\varepsilon>0, \quad a(0)=0, \quad \int_{0}^{1} \frac{a(r)}{r} \mathrm{~d} r<\infty .
$$

Then the smooth functions are not dense in the Sobolev space determined by the energy $\int|\nabla u|^{2} \rho \mathrm{d} x$. The convergence of the Dini integral in (2.6) gives us exactly (2.5). In this case, it is easy to show that the codimension of $H$ in $W$ equals one [25]. Condition (2.6) is satisfied, for example, with $a(r)=\left(\ln \frac{1}{r}\right)^{-1-\varepsilon}, \varepsilon>0$.

Special mention should be given to the case where the exponent $p(x)$ is smooth everywhere except for one singular point. We cite an example from the paper [21. In the disk $B=\{|x|<1\} \subset \mathbb{R}^{n}$, let an exponent $p(\cdot)$ smooth outside of the origin be given. Then $H=W$ whenever

$$
p(x) \leq n+w(|x|), \quad w(r) \leq C r^{\frac{w(r)}{n-1}},
$$

or

$$
p(0)>n, \quad|p(x)-p(0)| \leq w(|x|), \quad w(r) \leq C r^{\frac{2 n w(r)}{p^{2}(0)-w^{2}(r)}} .
$$

First, we note that it is suffices to establish the existence of approximation for bounded functions, because the bounded functions are dense in the Sobolev space. For a parameter $\delta \in(0,1)$, we define a cut-off function

$$
\eta_{t}(x)=\eta_{t}(|x|)= \begin{cases}1 & \text { if } t \leq|x| \leq 1 \\ \frac{\ln \frac{|x|}{t}}{\ln \frac{1}{\delta}}+1 & \text { if } t \delta \leq|x| \leq t \\ 0 & \text { if } 0 \leq|x| \leq t \delta .\end{cases}
$$

Suppose that (2.7) is true. Put $u_{t}=\eta_{t} u$. Since the exponent $p(\cdot)$ is smooth outside zero, we have $u_{t} \in H$. We show that

$$
\nabla u_{t}=u \nabla \eta_{t}+\eta_{t} \nabla u \rightarrow \nabla u \text { in } L^{p(\cdot)}(B) \text { as } t \rightarrow 0 .
$$

For that, since $u$ is bounded, it suffices to cheek the uniform boundedness of the sequence $\nabla \eta_{t}$ in $L^{p(\cdot)}(B)$. Using (2.7), we obtain

$$
\int\left|\nabla \eta_{t}\right|^{p} \mathrm{~d} x \leq|B|+\int\left|\nabla \eta_{t}\right|^{n+w(t)} \mathrm{d} x \leq|B|+\frac{1}{\left(\ln \frac{1}{\delta}\right)^{n+w(t)} w(t)(t \delta)^{w(t)}} .
$$

For $t$ fixed, the denominator of the last fraction attains its maximum when $\delta$ is determined by the equation

$$
\ln \frac{1}{\delta}=\frac{n+w(t)}{w(t)}
$$


Substituting this value, we obtain

$$
\int\left|\nabla \eta_{t}\right|^{p} \mathrm{~d} x \leq|B|+\frac{1}{t^{w(t)}\left(\frac{n+w(t)}{w(t)}\right)^{n+w(t)} e^{-(n+w(t))} w(t)} \leq|B|+C \frac{w(t)^{n-1}}{t^{w(t)}} .
$$

By (2.7), the right-hand side of this inequality is bounded.

Note that example (2.4) shows that the exponent $1 /(n-1)$ in (2.7) cannot be improved. Indeed, in that case we have $n=2$, and condition (2.7) turns into $w(r) \leq C r^{w(r)}$. Substituting $w(r)=k \chi(r)$ from (2.4), we obtain $k \frac{\ln \ln \frac{1}{r}}{\ln \frac{1}{r}} \leq C\left(\ln \frac{1}{r}\right)^{-k}$. Obviously, this relation holds for $k<1$. If we could replace $(n-1)=1$ here with $1+\varepsilon$, then (2.7) would also be satisfied for $1<k<1+\varepsilon$. But example (2.4) shows that $H \neq W$ in this case.

Turning to condition (2.8), we consider $u_{t}=(u-u(0)) \eta_{t}+u(0)$ as an approximation of $u$. As before, we verify that the sequence $(u-u(0)) \nabla \eta_{t}$ is bounded in $L^{p(\cdot)}(B)$. The Morrey inequality yields

$$
|u(x)-u(0)| \leq C|x|^{1-\frac{n}{p(0)-w(t)}}, \quad|x| \leq t .
$$

Hence,

$$
\int\left|\nabla \eta_{t}\right|^{p}|u-u(0)|^{p} \mathrm{~d} x \leq|B|+\frac{C}{\left(\ln \frac{1}{\delta}\right)^{p(0)+w(t)} w(t) t^{\frac{2 n w(t)}{p(0)-w(t)}}} .
$$

Now we choose

$$
\ln \frac{1}{\delta}=\frac{p(0)+w(t)}{2 n w(t)}
$$

with leads to

$$
\int\left|\nabla \eta_{t}\right|^{p}|u-u(0)|^{p} \mathrm{~d} x \leq|B|+C \frac{w(t)^{p(0)+w(t)}}{t^{\frac{2 n w(t)}{p(0)-w(t)}}} .
$$

Then condition (2.8) gives us the uniform boundedness of the right-hand side.

On the plane, condition (2.8) can be replaced with a simpler condition $p(x) \geq 2$. By a duality argument, that fact follows from the regularity of the exponent $p(x) \leq 2$ on the plane. The latter fact is implied by condition (2.7) for $w(t) \equiv 0$.

Now we turn our attention to the effects arising in the presence of a weight that degenerates on a "thin" set. Suppose that the weight $\rho$ degenerates only on a closed bounded set $F \subset \Omega$, i.e.,

$$
0<c_{1}(\varepsilon) \leq \rho(x) \leq c_{2}(\varepsilon)<\infty, \quad x \notin F_{\varepsilon}:=\{x \in \Omega: \operatorname{dist}(x, F) \leq \varepsilon\}, \quad \varepsilon>0 .
$$

The capacity of a set $F$ is introduced by the formula

$$
\operatorname{cap}_{p} F=\inf \left\{\int_{\Omega}|\nabla u|^{p(x)} \mathrm{d} x: u \in C_{0}^{\infty}(\Omega), u=1 \text { in the vicinity of } F\right\} .
$$

It is known (see, e.g., [26]) that for the variable exponent capacity inherits the usual properties. In particular, the set of zero capacity has zero measure. We show that the weight is regular whenever

$$
\operatorname{cap}_{p} F=0, \quad \rho(x) \leq \frac{\text { const }}{\operatorname{cap}_{p} F_{\varepsilon}}, \quad x \in \Omega \backslash F_{\varepsilon} .
$$

For example, if $F=\{0\}$ and $p=$ const $\leq n$, then condition (2.9) takes the form

$$
\sup _{|x|>\varepsilon} \rho(x) \leq C \begin{cases}\ln \frac{1}{\varepsilon} & \text { if } \quad n=p \\ \varepsilon^{p-n} & \text { if } \quad p<n .\end{cases}
$$

If $p=$ const $>n$, then the capacity of the isolated point is positive.

So, the problem is to approximate a function $u \in W$ with smooth functions in the norm of $W$ under condition (2.9). Without loss of generality we may assume that $\sup _{\Omega}|u| \leq 1$. 
By the definition of capacity, there exists a sequence of functions $\theta_{\varepsilon} \in C^{\infty}(\Omega)$ such that $\theta_{\varepsilon}=0$ on $F_{\varepsilon}, 0 \leq \theta_{\varepsilon} \leq 1, \int_{\Omega}\left|\nabla \theta_{\varepsilon}\right|^{p(x)} \mathrm{d} x \leq 2 \operatorname{cap}_{p} F_{\varepsilon}$, and $\theta_{\varepsilon} \rightarrow 1$ a.e. in $\Omega$ as $\varepsilon \rightarrow 0$. Put $u_{\varepsilon}=u \theta_{\varepsilon}$. We estimate

$$
\int_{\Omega}\left|\nabla u_{\varepsilon}\right|^{p(x)} \rho \mathrm{d} x \leq \int_{\Omega}|\nabla u|^{p(x)} \rho \mathrm{d} x+2 \sup _{\Omega \backslash F_{\varepsilon}} \rho \cdot \operatorname{cap}_{p} F_{\varepsilon} \leq C<\infty .
$$

Thus, we may assume that $u_{\varepsilon} \rightarrow u, \nabla u_{\varepsilon} \rightarrow \nabla u$ weakly in $L^{p(\cdot)}(\Omega, \rho \mathrm{d} x)$. Since $\operatorname{supp} u_{\varepsilon}$ lies in the set $\Omega \backslash F_{\varepsilon}$, where the weight is separated away from zero and infinity, we may replace $u_{\varepsilon}$ with functions smooth on the closure of the domain. To obtain strong convergence, it remains to use the Mazur lemma. For example, this implies that a weight is regular if it is bounded and degenerates only on a set of zero capacity. A similar proof of the regularity of a weight is available under the condition

$$
\text { meas } F=0, \quad \operatorname{cap}_{p}(F, \rho)=0,
$$

where

$$
\operatorname{cap}_{p}(F, \rho)=\inf \left\{\int_{\Omega}|\nabla u|^{p(x)} \rho \mathrm{d} x: u \in C_{0}^{\infty}(\Omega), u=1 \text { in a neighborhood of } F\right\} .
$$

We compare condition (2.10) (or (2.11)) with the statement of Theorem 1, Let $p=2$, and suppose that the weight $\rho$ in the disk $\Omega=\{|x|<1\} \subset \mathbb{R}^{2}$ satisfies.

$$
1 \leq \rho \leq C\left(\ln \frac{2}{|x|}\right)^{\gamma} .
$$

It is easy to verify that the $\rho$-capacity of the point $\{0\}$ is positive for $\gamma>1$. Indeed, for any $u \in C_{0}^{\infty}(\Omega)$ such that $u(0)=1$, we have

$$
1 \leq \int_{0}^{1}\left|u_{r}(r, \theta)\right| \mathrm{d} r
$$

Integrating with respect to the angle and using the Hölder inequality, for $\gamma>1$ we obtain

$$
\begin{aligned}
2 \pi & \leq \int_{0}^{2 \pi} \mathrm{d} \theta \int_{0}^{1}\left|u_{r}(r, \theta)\right| \mathrm{d} r \\
& \leq\left(\int_{\Omega}|\nabla u|^{2}\left(\ln \frac{2}{|x|}\right)^{\gamma} \mathrm{d} x\right)^{1 / 2}\left(2 \pi \int_{0}^{1}\left(\frac{2}{r}\right)^{-\gamma} \frac{d r}{r}\right)^{1 / 2} .
\end{aligned}
$$

Therefore,

$$
\int_{\Omega}|\nabla u|^{2}\left(\ln \frac{2}{|x|}\right)^{\gamma} \mathrm{d} x \geq C=C(n, \gamma)>0 .
$$

Thus, for $\gamma>1$ the class $C_{0}^{\infty}(\Omega \backslash\{0\})$ is not dense in $W$. On the other hand, it is easily seen that

$$
\lim _{n \rightarrow \infty} \frac{1}{n}\left[\int_{0}^{1} r\left(\ln \frac{1}{r}\right)^{n} \mathrm{~d} r\right]^{1 / n}=\frac{1}{2 e}
$$

Hence, if $\rho$ satisfies (2.12), then

$$
\lim _{n \rightarrow \infty} \frac{\left(\int_{\Omega} \rho^{n} \mathrm{~d} x\right)^{1 / n}}{n^{2}}<\infty
$$

for $\gamma \leq 2$. Thus, by Theorem 1 , the $C^{\infty}(\Omega)$ functions are dense in $W$.

On the other hand, in the same domain we can consider the weight

$$
\rho(x)= \begin{cases}\left(\ln \frac{2}{|x|}\right)^{\gamma} & \text { if } \quad x_{1} x_{2}>0 \\ \left(\ln \frac{2}{|x|}\right)^{-\gamma} & \text { if } \quad x_{1} x_{2}<0\end{cases}
$$


For $\gamma>1$ this weight is irregular, which follows from the example above (put $a(r)=$ $\left(\ln \frac{2}{r}\right)^{-\gamma}$ in (2.6)). For $\gamma \leq 1$, from Theorem 1 it follows that this weight is regular. This example shows that the condition of Theorem 1 is sharp - the exponent 2 in the denominator cannot be replaced with $2+\varepsilon$ for an arbitrarily small $\varepsilon$.

In the two-dimensional case, the class of conditions sufficient for the weight regularity is wider. We show that if the weight $\rho$ is regular and $\rho^{-p^{\prime} / p} \in L^{1}(\Omega)$, then the weight $\rho^{-p^{\prime} / p}$ is regular for the exponent $p^{\prime}$, i.e., $H\left(\Omega, p^{\prime}(\cdot), \rho^{-p^{\prime} / p}\right)=W\left(\Omega, p^{\prime}(\cdot), \rho^{-p^{\prime} / p}\right)$. Suppose the contrary. Let the weight $\rho^{1 /(1-p)}$ be irregular for the weighted Sobolev space with exponent $p^{\prime}(\cdot)$, i.e., $H\left(\Omega, p^{\prime}(\cdot), \rho^{-p^{\prime} / p}\right) \neq W\left(\Omega, p^{\prime}(\cdot), \rho^{-p^{\prime} / p}\right)$. Then there exists $u \in W\left(\Omega, p^{\prime}(\cdot), \rho^{-p^{\prime} / p}\right)$ such that $\nabla u$ does not belong to the closure of smooth vectorvalued functions in the gradient norm in $\left(L^{p^{\prime}(\cdot)}\left(\Omega, \rho^{-p^{\prime} / p} \mathrm{~d} x\right)\right)^{2}$. Hence, there exists $\mathbf{g} \in\left(L^{p(\cdot)}(\Omega, \rho \mathrm{d} x)\right)^{2}$ such that

$$
\int_{\Omega} \mathbf{g} \nabla \varphi \mathrm{d} x=0 \quad \text { for all } \quad \varphi \in C_{0}^{\infty}(\Omega), \quad \int_{\Omega} \mathbf{g} \nabla u \mathrm{~d} x \neq 0 .
$$

Thus, vector $\mathbf{g}$ is solenoidal. Since the vector $\left\{-g_{2}, g_{1}\right\}$ is potential, we can write

$$
\mathbf{g}=\nabla^{*} v=\left\{-\frac{\partial v}{\partial x_{2}}, \frac{\partial v}{\partial x_{1}}\right\}, \quad v \in W^{1,1}(\Omega) .
$$

Since the weight $\rho$ is regular, there exists a sequence $v_{\varepsilon} \in C^{\infty}(\Omega)$ such that $\nabla^{*} v_{\varepsilon} \rightarrow \mathbf{g}$ in $\left(L^{p(\cdot)}(\Omega, \rho \mathrm{d} x)\right)^{2}$. Since

$$
0=\int \nabla^{*} v_{\varepsilon} \nabla u \mathrm{~d} x \rightarrow \int \mathrm{g} \nabla u \mathrm{~d} x
$$

we arrive at a contradiction.

\section{§3. LiPSCHITZ TRUNCATIONS METHOD}

Denote

$$
d(x)=\operatorname{dist}(x, \partial \Omega), \quad g=M(\nabla u),
$$

where $M$ is the Hardy-Littlewood maximal operator:

$$
M f(x)=\sup _{x \in B} \frac{1}{|B|} \int_{B}|f| \mathrm{d} x \text { for } f \in L_{\text {loc }}^{1}\left(\mathbb{R}^{n}\right),
$$

with the supremum taken over all balls containing $x$. Here and in what follows we assume that $u \in W_{0}^{1,1}(\Omega)$ is extended by zero to $\mathbb{R}^{n} \backslash \Omega$.

The following fact is known: for almost all points $x, y \in \Omega$ we have

$$
|u(x)-u(y)| \leq C\{M(\nabla u)(x)+M(\nabla u)(y)\}|x-y| .
$$

This inequality is a direct consequence of the Poincaré inequality and the following simple estimate: for a.e. $x \in B$ we have

$$
\left|u(x)-u_{B}\right| \leq C \int_{B} \frac{|\nabla u(y)|}{|x-y|^{n-1}} \mathrm{~d} y \leq C R M(\nabla u)(x), \quad u_{B}=\frac{1}{|B|} \int_{B} u(y) \mathrm{d} y,
$$

which is valid for every ball $B \subset \mathbb{R}^{n}$ of radius $R$. To obtain (3.1), it suffices to consider the ball $B$ in which the points $x, y$ are endpoints of a diameter.

Moreover, in a Lipschitz domain we have

$$
|u(x)| \leq C d(x) M(\nabla u)(x), \quad u \in W_{0}^{1,1}(\Omega), \text { for a.e. } x \in \Omega .
$$

In fact, to have (3.2) it suffices to know that for every ball $B$ centered on the boundary $\partial \Omega$ the inequality $|B \backslash \Omega| \geq \gamma|B|$ is fulfilled for $\gamma=$ const $>0$. We provide the proof of 
this fact. Consider the ball $B=B\left(x_{0}, R\right)$ centered on the boundary of $\Omega$. As before, using the Poincaré inequality, for every pair of points $x \in B \cap \Omega$ and $y \in B \backslash \Omega$ we obtain

$$
\begin{aligned}
|u(x)| & =|u(x)-u(y)| \leq\left|u(x)-u_{B}\right|+\left|u(y)-u_{B}\right| \\
& \leq C \int_{B} \frac{|\nabla u(z)|}{|x-z|^{n-1}} \mathrm{~d} z+C \int_{B} \frac{|\nabla u(z)|}{|y-z|^{n-1}} \mathrm{~d} z .
\end{aligned}
$$

The first integral is estimated as before. We show that

$$
\inf _{y \in B \backslash \Omega} \int_{B} \frac{|\nabla u(z)|}{|y-z|^{n-1}} \mathrm{~d} z \leq C \int_{B} \frac{|\nabla u(z)|}{|x-z|^{n-1}} \mathrm{~d} z .
$$

For this, we consider the quantity

$$
\begin{aligned}
\frac{1}{|B \backslash \Omega|} \int_{B \backslash \Omega} \mathrm{d} y \int_{B} \frac{|\nabla u(z)|}{|y-z|^{n-1}} \mathrm{~d} z & =\int_{B}|\nabla u(z)| \mathrm{d} z \frac{1}{|B \backslash \Omega|} \int_{B \backslash \Omega}|y-z|^{1-n} \mathrm{~d} y \\
& \leq C \int_{B} \frac{|\nabla u(z)|}{|x-z|^{n-1}} \mathrm{~d} z .
\end{aligned}
$$

Here we have used the trivial inequality

Denote

$$
\frac{1}{B\left(x_{0}, R\right)} \int_{B\left(x_{0}, R\right)}|y-z|^{1-n} \mathrm{~d} z \leq C R^{1-n} \leq C|x-z|^{1-n} .
$$

$$
F_{\lambda}=\Omega \cap\{g \leq \lambda\} .
$$

The above estimates show that the restriction of $u$ to $F_{\lambda}$, extended by zero to $\left(\mathbb{R}^{n} \backslash \Omega\right)$, is a Lipschitz function with Lipschitz constant $C \lambda$ :

$$
|u(x)-u(y)| \leq C \lambda|x-y| \text { for } x, y \in F_{\lambda} \cup\left(\mathbb{R}^{n} \backslash \Omega\right) .
$$

Using the McShane theorem, we obtain a Lipschitz function $u_{\lambda}$ with Lipschitz constant $C \lambda$ that coincides with $u$ on $F_{\lambda}$ and vanishes outside $\Omega$.

We show that $u_{\lambda} \in H$, i.e., $u_{\lambda}$ can be approximated in the norm of $W$ by functions of class $C_{0}^{\infty}(\Omega)$.

Lemma. Suppose that a Lipschitz function $v$ on the closure of the domain $\Omega$ vanishes on the boundary $\partial \Omega$. Then $v \in H$.

Proof. We may assume that $v \geq 0$, otherwise we consider separately the positive and the negative part of $v$. First, we replace $v$ with $v_{\delta}=\max (v, \delta)-\delta$; then we apply a standard mollification by convolution with a smooth kernel. We obtain functions $v_{\delta, \varepsilon}=T_{\varepsilon} v_{\delta}$. At the same time, the $\nabla v_{\delta, \varepsilon}$ converge to $\nabla v_{\delta}$ almost everywhere and their absolute values are uniformly bounded by $\sup |\nabla v|$. Thus, they converge in the energy norm. The convergence of $v_{\delta}$ to $v$ as $\delta \rightarrow 0$ in the energy norm follows from the integrability of the weight $\rho$.

\section{§4. Local Muckenhoupt Classes in the Case of a variable exponent}

Definition. We say that a nonnegative locally integrable function $\omega$ belongs to the class $A_{p(\cdot)}(\Omega)$ if the set of zeros of $\omega$ has zero Lebesgue measure and

$$
\|w\|_{A_{p(\cdot)}(\Omega)}:=\sup _{Q} \int_{Q}\left(\frac{\omega(Q)}{|Q|^{p} \omega}\right)^{\frac{1}{p-1}} \mathrm{~d} x<\infty,
$$

where the supremum is taken over all cubes $Q \subset \Omega$ with faces parallel to coordinate hyperplanes. Here and in the sequel we use the notation

$$
\omega(E)=\int_{E} \omega \mathrm{d} x .
$$


It is easy to verify that for a constant exponent $p(\cdot) \equiv$ const this condition is precisely the same as the classical condition ensuring that the weight belongs to the Muckenhoupt class $A_{p}$ :

$$
\sup _{Q} \frac{1}{|Q|} \int_{Q} \omega \mathrm{d} x\left(\frac{1}{|Q|} \int_{Q} \omega^{\frac{1}{1-p}} \mathrm{~d} x\right)^{p-1}<\infty .
$$

We explain the origin of condition (4.1). The following question arises naturally: what condition should be imposed on the weight in the case of a variable exponent in order that the implication

$$
\frac{1}{|Q|} \int_{Q} f \mathrm{~d} x \geq 1 \Rightarrow \frac{1}{\omega(Q)} \int_{Q}|f|^{p(x)} \omega \mathrm{d} x>\gamma, \quad \gamma>0
$$

be true uniformly for all cubes $Q$ and functions $f$ ? In the case of a constant exponent $p>1$, condition (4.3) is precisely the Muckenhoupt condition. Indeed, by the Hölder inequality and the Muckenhoupt condition (4.2), we have

$$
\left(\frac{1}{|Q|} \int_{Q}|f| \mathrm{d} x\right)^{p} \leq \frac{1}{|Q|} \int_{Q}|f|^{p} \omega \mathrm{d} x\left(\frac{1}{|Q|} \int_{Q} \omega^{\frac{1}{1-p}} \mathrm{~d} x\right)^{p-1} \leq C \frac{1}{\omega(Q)} \int_{Q}|f|^{p} \omega \mathrm{d} x .
$$

In the case of a variable exponent, first we use the Hölder inequality for $L^{p(\cdot)}$ norms:

$$
\begin{aligned}
|Q| \leq \int_{Q}|f| \mathrm{d} x & \leq 2\left\|f \omega^{1 / p} \omega(Q)^{-1 / p}\right\|_{L^{p}(Q)}\left\|\omega^{-1 / p} \omega(Q)^{1 / p}\right\|_{L^{p^{\prime}}(Q)} \\
& =2\left\|f \omega(Q)^{-1 / p}\right\|_{L^{p}(Q, \omega \mathrm{d} x)}\left\|\omega^{-1 / p} \omega(Q)^{1 / p}\right\|_{L^{p^{\prime}}(Q)} .
\end{aligned}
$$

Hence,

$$
\left\|f \omega(Q)^{-1 / p}\right\|_{L^{p}(Q, \omega \mathrm{d} x)} \geq \frac{|Q|}{2\left\|\omega^{-1 / p} \omega(Q)^{1 / p}\right\|_{L^{p^{\prime}}(Q)}} .
$$

If condition (4.1) is satisfied, then the definition of the norm shows that

$$
\left\|\omega^{-1 / p} \omega(Q)^{1 / p}\right\|_{L^{p^{\prime}}(Q)}<C|Q|,
$$

whence

$$
\left\|f \omega(Q)^{-1 / p}\right\|_{L^{p}(Q, \omega \mathrm{d} x)} \geq \gamma>0 .
$$

Thus, we obtain

$$
\frac{1}{\omega(Q)} \int_{Q}|f|^{p} \omega \mathrm{d} x \geq \gamma=\text { const }>0 .
$$

We formulate this in the following lemma.

Lemma. If $\omega \in A_{p(\cdot)}(\Omega)$, then there exists a constant $\gamma>0$ such that

$$
\frac{1}{|Q|} \int_{Q} f \mathrm{~d} x \geq 1 \Rightarrow \frac{1}{\omega(Q)} \int_{Q}|f|^{p(x)} \omega \mathrm{d} x>\gamma
$$

for all cubes $Q \subset \Omega$.

Lemma. Let $\omega \in A_{p(\cdot)}(\Omega)$. Then for any cube $Q \subset \Omega$ and any measurable set $E \subset Q$ we have

$$
\gamma\left(\frac{|E|}{|Q|}\right)^{\beta}<\frac{\omega(E)}{\omega(Q)}<\gamma_{1}\left(\frac{|E|}{|Q|}\right)^{\beta_{1}}
$$


Proof. Indeed, let $E \subset Q$. Denote $f=\chi_{E}|Q| /|E|$. Obviously,

$$
\frac{1}{|Q|} \int_{Q} f \mathrm{~d} x=1
$$

Hence,

$$
\left(\frac{|Q|}{|E|}\right)^{\beta} \omega(E) \geq \int_{E}\left(\frac{|Q|}{|E|}\right)^{p} \omega \mathrm{d} x=\int_{Q}|f|^{p} \omega \mathrm{d} x>\gamma \omega(Q),
$$

where $\gamma$ is a constant occurring in (4.4). Thus,

$$
\frac{\omega(E)}{\omega(Q)}>\gamma\left(\frac{|E|}{|Q|}\right)^{\beta}
$$

The classical methods of harmonic analysis (see [7, Chapter 5 and, e.g., Proposition 4 on pp. 202-203] show that an opposite estimate is also valid:

$$
\frac{\omega(E)}{\omega(Q)} \leq \gamma_{1}\left(\frac{|E|}{|Q|}\right)^{\beta_{1}}, \quad \gamma_{1}, \beta_{1}>0
$$

Lemma. If the exponent $q(\cdot)$ satisfies the logarithmic continuity condition (1.4), then there exists a constant $C>0$ such that for every cube $Q \subset \Omega$ we have

$$
\omega(Q)^{q(x)-q(y)} \leq C \quad \text { for all } \quad x, y \in Q .
$$

Consequently, there exist constants $c_{1}, c_{2}>0$ such that

$$
c_{1} \omega(Q)^{1 / p(x)} \leq\left\|\omega^{1 / p}\right\|_{L^{p}(Q)} \leq c_{2} \omega(Q)^{1 / p(x)} \quad \text { for all } \quad x \in Q .
$$

Proof. Using the Lipschitz property of the boundary, it is easy to prove that there exist constants $C_{1}, C_{2}, \gamma_{1}, \gamma_{2}>0$ such that

$$
C_{1}|Q|^{\gamma_{1}} \leq \omega(Q) \leq C_{2}|Q|^{\gamma_{2}}
$$

for every cube $Q \subset \Omega$. Indeed, for sufficiently large cubes this fact is obvious because the domain is bounded. If the given cube is contained inside a sufficiently large cube, then the assertion follows from the preceding lemma. But if the cube in question lies sufficiently close to the boundary, then it could be connected by a chain of cubes with a sufficiently large cube so that the edges of the adjacent cubes be comparable, and the number of cubes in the chain be comparable to the ratio of the edges of the first and the last cubes. At the last step we use the Lipschitz property of the boundary. Here it is convenient to imagine the cube in question as positioned near the vertex of an acute angle pointed outside the domain.

Since the domain is bounded, it suffices to verify property (4.6) for all cubes with $|Q|<1$. For such cubes, the logarithmic Hölder condition implies that

$$
\omega(Q)^{q(x)-q(y)} \leq C|Q|^{-C \operatorname{osc}_{Q} q}=C \exp \left(\ln |Q| \frac{C}{\ln |Q|^{-1}}\right) \leq C .
$$

The following lemma expresses a duality property of the Muckenhoupt classes introduced above.

Lemma. If $\omega \in A_{p(\cdot)}(\Omega)$, then $\omega^{-p^{\prime} / p} \in A_{p^{\prime}(\cdot)}(\Omega)$. In particular, for every exponent $q(\cdot)$ satisfying the logarithmic Hölder condition (1.4), the estimate

$$
\left(\omega^{\frac{1}{1-p}}(Q)\right)^{q(x)-q(y)} \leq C, \quad x, y \in Q,
$$

holds true for every cube $Q \subset \Omega$. Consequently, there exist constants $c_{1}, c_{2}>0$ such that

$$
c_{1}\left(\omega^{\frac{1}{1-p}}(Q)\right)^{1 / p^{\prime}(x)} \leq\left\|\omega^{-1 / p}\right\|_{L^{p^{\prime}}(Q)} \leq c_{2}\left(\omega^{\frac{1}{1-p}}(Q)\right)^{1 / p^{\prime}(x)}, \quad x \in Q .
$$


Proof. We recall that the relation $\omega \in A_{p(\cdot)}(\Omega)$ is equivalent to

$$
\sup _{Q} \int_{Q}\left(\frac{\omega(Q)}{|Q|^{p} \omega}\right)^{\frac{1}{p-1}} \mathrm{~d} x<\infty .
$$

Using the preceding lemma, we obtain

$$
\omega^{\frac{1}{1-p}}(Q) \leq C \frac{|Q|^{p^{\prime}(x)}}{\omega(Q)^{\frac{1}{p(x)-1}}}, \quad x \in Q,
$$

for every cube $Q \subset \Omega$. Using this estimate, we obtain

$$
\int_{Q} \frac{\left(\omega^{\frac{1}{1-p}}(Q)\right)^{p-1} \omega \mathrm{d} x}{|Q|^{p}} \leq C \int_{Q} \frac{|Q|^{p}}{|Q|^{p} \omega(Q)} \omega \mathrm{d} x \leq C
$$

for every cube $Q \subset \Omega$, which means precisely that $\omega^{-p^{\prime} / p} \in A_{p^{\prime}(\cdot)}(\Omega)$.

We show that the classes $A_{p(\cdot)}(\Omega)$ grow as $p$ increases.

Lemma. If two exponents $p(\cdot)$ and $q(\cdot)$ satisfy the logarithmic condition and $p \leq q$, then $A_{p(\cdot)}(\Omega) \subset A_{q(\cdot)}(\Omega)$.

Proof. Using the definition (4.1) and the Young inequality, we obtain

$$
\begin{aligned}
\|\omega\|_{A_{q(\cdot)}(\Omega)} & =\sup _{Q} \int_{Q}\left(\frac{\omega(Q)}{|Q|^{q} \omega}\right)^{\frac{1}{q-1}} \mathrm{~d} x \\
& \leq \sup _{Q} \int_{Q}\left(\frac{\omega(Q)}{|Q|^{p} \omega}\right)^{\frac{1}{p-1}} \mathrm{~d} x+\sup _{Q} \int_{Q} \frac{q-p}{q-1}\left(\frac{p-1}{q-1}\right)^{\frac{p-1}{q-p}} \frac{\mathrm{d} x}{|Q|} \\
& \leq\|\omega\|_{A_{p(\cdot)}(\Omega)}+1 .
\end{aligned}
$$

Now we prove the main theorem of this section.

Theorem 5. Let $\omega \in A_{p(\cdot)}(\Omega)$, and let the exponent $p(\cdot)$ satisfy the logarithmic condition (1.4). Then there exists a constant $\varepsilon>0$ such that $\omega \in A_{p(\cdot)-\varepsilon}(\Omega)$. In particular, for every cube or ball $Q \subset \Omega$ the implication

$$
\frac{1}{|Q|} \int_{Q}|f| \mathrm{d} x \geq 1 \Rightarrow \frac{1}{\omega(Q)} \int_{Q}|f|^{p(x)-\varepsilon} \omega \mathrm{d} x \geq \gamma
$$

is true for some $\gamma, \varepsilon>0$.

Proof. It suffices to verify condition (4.1) for sufficiently small cubes. By the preceding lemma, for a cube $Q \subset \Omega$ we have

$$
\omega \in A_{p_{+}(Q)}(Q), \quad p_{+}(Q)=\max _{x \in Q} p(x),
$$

and moreover,

$$
\|\omega\|_{A_{p_{+}(Q)}(Q)} \leq\|\omega\|_{A_{p(\cdot)}(\Omega)}+1=C_{0}
$$

The theory of Muckenhoupt weights provides the existence of constants $\varepsilon>0$ and $C_{1}$ such that on every cube $Q \subset \Omega$ we have

$$
\omega \in A_{p_{+}(Q)-2 \varepsilon}(Q), \quad\|\omega\|_{A_{p_{+}(Q)-2 \varepsilon}(Q)} \leq C_{1} .
$$

For sufficiently small cubes, $|Q|<c_{0}$, such that the oscillation of the exponent does not exceed $\varepsilon$, we obtain

$$
\|\omega\|_{A_{p_{-}(Q)-\varepsilon}(Q)} \leq C .
$$


By the monotonicity property of Muckenhoupt classes (see above), for sufficiently small cubes we get

$$
\|\omega\|_{A_{p(\cdot)-\varepsilon}(Q)} \leq C .
$$

Remark. Here we consider the Muckenhoupt classes in a bounded domain. In the entire space, the theory is more complicated. It requires additional assumptions of exponent stabilization at infinity, e.g., a logarithmic estimate. Such research was done in the papers [27, 28] and in the book 222. There, the Muckenhoupt class with variable exponent was introduced in several different ways. In [27] the class $A_{p(\cdot)}^{\dagger}$ was defined by the condition

$$
\left\|\omega \chi_{Q}\right\|_{1}\left\|\omega^{-1} \chi_{Q}\right\|_{p^{\prime}(\cdot) / p(\cdot)} \leq C|Q|^{p_{Q}}, \quad p_{Q}=\left(\frac{1}{|Q|} \int_{Q} p(x)^{-1} \mathrm{~d} x\right)^{-1} .
$$

Also there, another class $A_{p(\cdot)}^{\sharp}$ was defined by the condition

$$
\left\|\omega^{1 / p(\cdot)} \chi_{Q}\right\|_{p(\cdot)}\left\|\omega^{-1 / p(\cdot)} \chi_{Q}\right\|_{p^{\prime}(\cdot)} \leq C|Q| .
$$

In 28 it was proved that (4.8) and (4.9) can be replaced by the following natural definition of $A_{p(\cdot)}$ :

$$
\left\|\omega \chi_{Q}\right\|_{p(\cdot)}\left\|\omega^{-1} \chi_{Q}\right\|_{p^{\prime}(\cdot)} \leq C|Q|
$$

Under the logarithmic continuity condition on the exponent, supplemented by the logarithmic stabilization of the exponent at infinity, the classes $A_{p(\cdot)}^{\dagger}, A_{p(\cdot)}^{\sharp}$, and $A_{p(\cdot)}$ coincide, and the fact that the weight $\omega$ belongs to that class is equivalent to the boundedness of the Hardy-Littlewood maximal operator in $L^{p(\cdot)}\left(\mathbb{R}^{n}, \omega \mathrm{d} x\right)$. These matters are accounted in detail in the book [29]. Here a simple version of the theory is presented, sufficient for our objectives.

\section{$\S 5$. The MAin Result of The PAPER}

Theorem 6. Suppose that the exponent $p(\cdot)$ satisfies conditions (1.1) and (1.4), the weight $\rho$ has the form $\rho=\omega \omega_{0}$ with $\omega_{0} \in A_{p(\cdot)}(\Omega)$, and the following condition is satisfied:

$$
\liminf _{t \rightarrow \infty}\left(\int_{\Omega} \omega^{-t} \omega_{0} \mathrm{~d} x\right)^{1 / t}\left(\int_{\Omega}\left(t^{-p(x)} \omega\right)^{t} \omega_{0} \mathrm{~d} x\right)^{1 / t}<\infty .
$$

Then the weight $\rho$ is regular.

In this theorem the logarithmic condition is essential. It is necessary for obtaining the properties of Muckenhoupt classes with variable exponent.

Proof. We use the notation $\mathrm{d} \mu=\omega_{0} \mathrm{~d} x$. Obviously, $H$ is a subspace of $W$. Suppose that $H$ does not coincide with $W$. Then there exists a nonzero functional $f \in W^{*}$ that vanishes on $H$, i.e.,

$$
\langle f, \varphi\rangle=0 \text { for all } \varphi \in H
$$

Using $f$, we construct $u \in W$ that solves the equation

$$
-\operatorname{div}\left(\rho|\nabla u|^{p-2} \nabla u\right)=f
$$

i.e.,

$$
\int|\nabla u|^{p-2} \nabla u \nabla \varphi \rho \mathrm{d} x=\langle f, \varphi\rangle, \quad \varphi \in W
$$

We find such a solution by the direct variational method, by minimizing the functional

$$
\mathbf{F}[u]=\int_{\Omega} \frac{|\nabla u|^{p(x)}}{p(x)} \rho \mathrm{d} x-\langle f, u\rangle \rightarrow \inf , \quad u \in W .
$$


The existence of a minimizer follows from the classical theorem of variational calculus for convex integrands (Tonelli's theorem). The minimizing function is the desired solution of (5.2).

We could also refer to the theory of monotone operators. It suffices to consider the monotone nonlinear operator $\mathcal{A}: W \rightarrow W^{*}$ defined by

$$
\langle\mathcal{A} u, \varphi\rangle=\int_{\Omega}|\nabla u|^{p-2} \nabla u \nabla \varphi \rho \mathrm{d} x, \quad \varphi \in W .
$$

It is easy to verify that the operator $\mathcal{A}$ is monotone, coercive, and semicontinuous. Unique solvability in $W$ follows from the classical theorem for monotone operators. It is easy to show that the resulting solution is a unique minimizer of the functional in (5.3).

By the choice of $f$ we have

$$
\int|\nabla u|^{p-2} \nabla u \nabla \varphi \rho \mathrm{d} x=0, \quad \varphi \in H .
$$

If we take the Lipschitz truncation $u_{\lambda}$ constructed in $\S 3$ as a test function in (5.4), we obtain

$$
\int_{\Omega}|\nabla u|^{p-2} \nabla u \nabla u_{\lambda} \rho \mathrm{d} x=0
$$

Denote

$$
F_{\lambda}=\{g \leq \lambda\} \cap \Omega, \quad g=M(\nabla u) .
$$

Splitting the integral in (5.5) into two parts, over $F_{\lambda}$ and over $\Omega \backslash F_{\lambda}$, we obtain

$$
\int_{F_{\lambda}}|\nabla u|^{p} \rho \mathrm{d} x=-\int_{\Omega \backslash F_{\lambda}}|\nabla u|^{p-2} \nabla u \nabla u_{\lambda} \rho \mathrm{d} x \leq C \lambda \int_{\Omega \backslash F_{\lambda}}|\nabla u|^{p-1} \rho \mathrm{d} x .
$$

Thus, we obtain the following fundamental estimate:

$$
\int_{\{g \leq \lambda\}}|\nabla u|^{p} \rho \mathrm{d} x \leq C \lambda \int_{\{g>\lambda\}}|\nabla u|^{p-1} \rho \mathrm{d} x .
$$

We multiply inequality (5.7) by $\varepsilon \lambda^{-1-\varepsilon}$ and integrate with respect to $\lambda$ from $K$ to infinity, where $K \geq 1$ is a number to be chosen later. Using the Fubini theorem, we obtain

$$
\begin{aligned}
\int_{K}^{\infty} \varepsilon \lambda^{-1-\varepsilon} \int_{g \leq \lambda}|\nabla u|^{p} \rho \mathrm{d} x \mathrm{~d} \lambda & =\int_{\Omega}|\nabla u|^{p} \rho \int_{\max \{g, K\}}^{\infty} \varepsilon \lambda^{-1-\varepsilon} \mathrm{d} \lambda \mathrm{d} x \\
& =\int_{\Omega} \max (g, K)^{-\varepsilon}|\nabla u|^{p} \rho \mathrm{d} x \\
\int_{K}^{\infty} \varepsilon \lambda^{-\varepsilon} \int_{g>\lambda}|\nabla u|^{p-1} \rho \mathrm{d} x \mathrm{~d} \lambda & =\int_{\Omega}|\nabla u|^{p-1} \rho\left(\int_{K}^{g} \varepsilon \lambda^{-\varepsilon} \mathrm{d} \lambda\right)_{+} \mathrm{d} x \\
& =\frac{\varepsilon}{1-\varepsilon} \int_{\Omega}\left(g^{1-\varepsilon}-K^{1-\varepsilon}\right)_{+}|\nabla u|^{p-1} \rho \mathrm{d} x .
\end{aligned}
$$

Thus,

$$
\int_{\Omega} \max (g, K)^{-\varepsilon}|\nabla u|^{p} \rho \mathrm{d} x \leq C \varepsilon \int_{\Omega}\left(g^{1-\varepsilon}-K^{1-\varepsilon}\right)_{+}|\nabla u|^{p-1} \rho \mathrm{d} x .
$$

The case of a constant exponent. In this case we can choose $K=0$. Accordingly, from (5.8) we obtain

$$
\begin{aligned}
\int_{\Omega} g^{-\varepsilon}|\nabla u|^{p} \rho \mathrm{d} x & \leq C \varepsilon \int_{\Omega} g^{1-\varepsilon}|\nabla u|^{p-1} \rho \mathrm{d} x \\
& \leq C \varepsilon\left(\int_{\Omega}|\nabla u|^{p} \rho \mathrm{d} x\right)^{\frac{p-1}{p}}\left(\int_{\Omega} g^{p(1-\varepsilon)} \rho \mathrm{d} x\right)^{\frac{1}{p}} .
\end{aligned}
$$


In what follows, we use the notation

$$
A=\left(\int_{\Omega}|\nabla u|^{p} \rho \mathrm{d} x\right)^{\frac{1}{p}}
$$

and let $\|\cdot\|_{q}$ denote the $L^{q}(\Omega, \mathrm{d} \mu)$ norm. Since $\omega_{0} \in A_{p}$, the maximal operator is bounded on $L^{p}\left(\mathbb{R}^{n}, \mathrm{~d} \mu\right)$. By the self-improvement property of the Muckenhoupt weights, for small $\delta<\delta_{0}\left(n, p, \omega_{0}\right)$ we also have

$$
\|M h\|_{p-\delta} \leq C\|h\|_{p-\delta}
$$

where $C=C\left(n, p, \omega_{0}\right)$.

For $\varepsilon^{\prime}>0$, the Hölder inequality $\left(1 / p+\varepsilon^{\prime} / p\left(p-\varepsilon^{\prime}\right)=1 /\left(p-\varepsilon^{\prime}\right)\right)$ yields

$$
\|\nabla u\|_{p-\varepsilon^{\prime}}=\left\|w^{-1 / p} w^{1 / p} \nabla u\right\|_{p-\varepsilon^{\prime}} \leq\left\|w^{1 / p} \nabla u\right\|_{p} \cdot\left\|w^{-1 / p}\right\|_{p\left(p-\varepsilon^{\prime}\right) / \varepsilon^{\prime}}=A\left\|w^{-1}\right\|_{\frac{p-\varepsilon^{\prime}}{\varepsilon^{\prime}}}^{1 / p} .
$$

Using (5.10), we obtain

$$
\|g\|_{p-\varepsilon^{\prime}} \leq C A\left\|w^{-1}\right\|_{\frac{p-\varepsilon^{\prime}}{\varepsilon^{\prime}}}^{1 / p}
$$

where the constant $C$ can be chosen the same for all sufficiently small $\varepsilon^{\prime}$.

Thus, for small $\varepsilon$, choosing $\varepsilon^{\prime} \in(0, p \varepsilon)$, we obtain

$$
\begin{aligned}
\int g^{-\varepsilon}|\nabla u|^{p} \rho \mathrm{d} x & \leq C A^{p-1} \frac{\varepsilon}{1-\varepsilon}\left(\int g^{p(1-\varepsilon)} \rho \mathrm{d} x\right)^{1 / p} \\
& \leq C A^{p-1} \varepsilon\left(\int g^{p-\varepsilon^{\prime}} \mathrm{d} \mu\right)^{\frac{1-\varepsilon}{p-\varepsilon^{\prime}}}\left(\int w^{\frac{p-\varepsilon^{\prime}}{p \varepsilon-\varepsilon^{\prime}}} \mathrm{d} \mu\right)^{\frac{p \varepsilon-\varepsilon^{\prime}}{p\left(p-\varepsilon^{\prime}\right)}} \\
& \leq C A^{p-\varepsilon}\left(\varepsilon^{p}\left\|w^{-1}\right\|_{\frac{p-\varepsilon^{\prime}}{\varepsilon^{\prime}}}^{1-\varepsilon}\|w\|_{\frac{p-\varepsilon^{\prime}}{p \varepsilon-\varepsilon^{\prime}}}\right)^{1 / p} .
\end{aligned}
$$

Next, let $\varepsilon=\tau \varepsilon^{\prime}$, where $\tau>1 / p$. Denote $t=\frac{p-\varepsilon^{\prime}}{p \varepsilon-\varepsilon^{\prime}}$. Obviously, $t \rightarrow \infty$ as $\varepsilon \rightarrow 0$. Passing to the limit as $\varepsilon \rightarrow 0$ (on the left-hand side we could use the Lebesgue dominated convergence theorem, though the monotone convergence theorem suffices), we get

$$
A^{p} \leq C A^{p}\left(\liminf _{t \rightarrow \infty} \frac{\left\|w^{-1}\right\|_{(p \tau-1) t}^{1-\varepsilon(t)}\|w\|_{t}}{t^{p}}\right)^{1 / p} \quad, \text { where } \varepsilon(t)=\frac{p \tau}{(p \tau-1) t+1} .
$$

If the limit in this estimate is small, we obtain $A=0$, and the claim follows. Thus, we have the density of smooth functions in a weighted Sobolev space provided

$$
\liminf _{t \rightarrow \infty} \frac{\left\|w^{-1}\right\|_{(p \tau-1) t}^{1-\varepsilon(t)}\|w\|_{t}}{t^{p}}<C_{0},
$$

where the positive constant $C_{0}$ depends only on the space dimension and on the constant in the estimate of the maximal operator in $L^{p}(\Omega, \mathrm{d} \mu)$. Fixing $\tau=2 / p$, we arrive at a symmetric form of the condition: the limit

$$
\liminf _{t \rightarrow \infty} \frac{\left\|w^{-1}\right\|_{t}^{1-\varepsilon(t)}\|w\|_{t}}{t^{p}}<C_{0}, \quad \varepsilon(t)=\frac{2}{t+1},
$$

must be small. Below we shall explain how to lift condition (5.11).

General case. Here we assume that $K>1$. Applying the Young inequality to the right-hand side of inequality (5.8), we obtain

$$
\int_{\Omega} \max (g, K)^{-\varepsilon}|\nabla u|^{p} \rho \mathrm{d} x \leq \frac{1}{2} \int_{\Omega}|\nabla u|^{p} \rho \mathrm{d} x+C \int_{\Omega} \varepsilon^{p}\left(g^{1-\varepsilon}-K^{1-\varepsilon}\right)_{+}^{p} \rho \mathrm{d} x .
$$


Denoting the last integral by $I_{\varepsilon}$, we estimate it in the following way:

$$
\begin{aligned}
I_{\varepsilon} & :=\int_{\Omega} \varepsilon^{p}\left(g^{1-\varepsilon}-K^{1-\varepsilon}\right)_{+}^{p} \rho \mathrm{d} x \\
& =\int_{K}^{\infty} \int_{\{g>\lambda\}} p(1-\varepsilon)\left(\lambda^{1-\varepsilon}-K^{1-\varepsilon}\right)_{+}^{p-1} \lambda^{-\varepsilon} \varepsilon^{p} \rho \mathrm{d} x \mathrm{~d} \lambda \\
& \leq \beta \int_{K}^{\infty} \int_{\{g>\lambda\}} \lambda^{p-1-p \varepsilon} \varepsilon^{p} \rho \mathrm{d} x \mathrm{~d} \lambda .
\end{aligned}
$$

To estimate the integral over the set $\{g>\lambda\}$, we use the standard technique based on the covering lemma. By the definition of the maximal operator, the set $E(\lambda):=\{g>\lambda\}$ can be covered by balls $B_{z}, z \in E_{\lambda}$, such that

$$
\frac{1}{\left|B_{z}\right|} \int_{B_{z}} f \mathrm{~d} x>\lambda, \text { where } f=|\nabla u| .
$$

The Besicovitch covering theorem allows us to find $N=N(n)$ countable collections of balls $B_{k, j}=B_{k, j}(\lambda), k=1, \ldots, N$, that do not overlap inside a collection (i.e., for a fixed index $k$ ), and, taken together, cover the set $E(\lambda)$. We denote $B_{k, j}^{\prime}=\Omega \cap B_{k, j}$. Thus,

$$
I_{2 \varepsilon} \leq C \int_{K}^{\infty} \sum_{k=1}^{N} \sum_{j=1}^{\infty} \int_{B_{k, j}^{\prime}} \lambda^{p-1-2 p \varepsilon} \varepsilon^{p} \rho \mathrm{d} x \mathrm{~d} \lambda .
$$

We have

$$
\left|B_{k, j}\right|<\frac{1}{\lambda} \int_{B_{k, j} \cap\{f>\lambda / 2\}} f \mathrm{~d} x+\frac{1}{\lambda} \int_{B_{k, j} \cap\{f \leq \lambda / 2\}} f \mathrm{~d} x \leq \frac{1}{\lambda} \int_{B_{k, j} \cap\{f>\lambda / 2\}} f \mathrm{~d} x+\frac{1}{2}\left|B_{k, j}\right|,
$$

whence

$$
\left|B_{k, j}\right| \leq \frac{2}{\lambda} \int_{B_{k, j} \cap\{f>\lambda / 2\}} f \mathrm{~d} x
$$

By the Young inequality,

$$
\left|B_{k, j}(\lambda)\right| \leq \frac{1}{\lambda}\left(\int_{\Omega} \frac{f^{p(x)}}{p(x)} \rho \mathrm{d} x+\int_{\Omega} \frac{\rho^{\frac{1}{1-p}}}{p^{\prime}(x)} \mathrm{d} x\right) \leq \frac{C}{\lambda} .
$$

Consequently, the radii of all balls that occur in the cover corresponding to the given $\lambda$ do not exceed $C \lambda^{-1 / n}$. The logarithmic condition (1.4) gives us the estimate

$$
\lambda^{p(x)-p(y)} \leq \exp \left(\ln \lambda \cdot \frac{C}{\ln \lambda}\right) \leq C, \quad x, y \in B_{k, j}(\lambda) .
$$

By Theorem 5, on the balls $B_{k, j}(\lambda)$ we have

$$
\int_{B k, j(\lambda) \cap\{f>\lambda / 2\}}\left(\frac{f}{\lambda}\right)^{p(x)-\delta} \mathrm{d} \mu \geq C \mu\left(B_{k, j}(\lambda)\right),
$$

where $\delta>0$ is one and the same for all balls considered. Denote

$$
p_{k, j, \lambda}=\max _{x \in B_{k, j}(\lambda)} p(x) .
$$


We set $\varepsilon^{\prime}=\varepsilon^{\prime \prime}=\varepsilon / 2$ and estimate the quantity

$$
\begin{aligned}
\int_{B_{k, j}(\lambda)} & \lambda^{p-1-2 p \varepsilon} \varepsilon^{p} \rho \mathrm{d} x \leq \lambda^{p_{k, j, \lambda}(1-2 \varepsilon)-1} \int_{B_{k, j}(\lambda)} \varepsilon^{p} \rho \mathrm{d} x \\
\leq & C \lambda^{p_{k, j, \lambda}-1-2 p_{k, j, \lambda} \varepsilon} \mu\left(B_{k, j}(\lambda)\right)^{1-\varepsilon^{\prime}}\left(\int_{B_{k, j}(\lambda)}\left(\varepsilon^{p} \omega\right)^{1 / \varepsilon^{\prime}} \mathrm{d} \mu\right)^{\varepsilon^{\prime}} \\
\leq & C \lambda^{p_{k, j, \lambda}(1-2 \varepsilon)-1}\left(\int_{B_{k, j}(\lambda) \cap\{f>\lambda / 2\}}(f / \lambda)^{p-\delta} \mathrm{d} \mu\right)^{1-\varepsilon^{\prime}}\left(\int_{B_{k, j}(\lambda)}\left(\varepsilon^{p} \omega\right)^{1 / \varepsilon^{\prime}} \mathrm{d} \mu\right)^{\varepsilon^{\prime}} \\
\leq & \left.C \lambda^{p_{k, j, \lambda}(1-2 \varepsilon)-1}\left(\int_{B_{k, j}(\lambda) \cap\{f>\lambda / 2\}}\left(\lambda^{-1} f\right)^{\left(1+\varepsilon^{\prime \prime}\right)(p-\delta)} \rho \mathrm{d} x\right)^{\frac{1-\varepsilon^{\prime}}{1+\varepsilon^{\prime \prime}}} \omega^{-1 / \varepsilon^{\prime \prime}} \mathrm{d} \mu\right)^{\frac{\varepsilon^{\prime \prime}\left(1-\varepsilon^{\prime}\right)}{1+\varepsilon^{\prime \prime}}}\left(\int_{B_{k, j}(\lambda)}\left(\varepsilon^{p} \omega\right)^{1 / \varepsilon^{\prime}} \mathrm{d} \mu\right)^{\varepsilon^{\prime}} \\
& \times\left(\int_{B_{k, j}(\lambda) \cap\{f>\lambda / 2\}} \lambda^{(p-1-p \varepsilon)\left(1+\varepsilon^{\prime \prime}\right) /\left(1-\varepsilon^{\prime}\right)}\left(\lambda^{-1} f\right)^{(p-\delta)\left(1+\varepsilon^{\prime \prime}\right)} \rho \mathrm{d} x\right)^{\frac{1-\varepsilon^{\prime}}{1+\varepsilon^{\prime \prime}}} \\
\leq & \left(\int_{B_{k, j}(\lambda) \cap\{f>\lambda / 2\}} \lambda^{-p \varepsilon\left(1+\varepsilon^{\prime \prime}\right) /\left(2 \varepsilon^{\prime \prime}\left(1-\varepsilon^{\prime}\right)\right)} \omega^{-1 / \varepsilon^{\prime \prime}} \mathrm{d} \mu\right)^{\frac{\varepsilon^{\prime \prime}\left(1-\varepsilon^{\prime}\right)}{1+\varepsilon^{\prime \prime}}} \\
& \times\left(\int_{B_{k, j}(\lambda) \cap\{f>\lambda / 2\}} \lambda^{-p \varepsilon /\left(2 \varepsilon^{\prime}\right)}\left(\varepsilon^{p} \omega\right)^{1 / \varepsilon^{\prime}} \mathrm{d} \mu\right)^{\varepsilon^{\prime}} \cdot \\
& \times\left(\int_{B_{k, j}(\lambda)}\right.
\end{aligned}
$$

Since

$$
(p-1-p \varepsilon)\left(1+\varepsilon^{\prime \prime}\right) /\left(1-\varepsilon^{\prime}\right) \leq p-1,
$$

we have

$$
\begin{aligned}
& \int_{B_{k, j}(\lambda)} \lambda^{p-1-2 p \varepsilon} \varepsilon^{p} \rho \mathrm{d} x \\
& \leq C\left(\int_{B_{k, j}(\lambda) \cap\{f>\lambda / 2\}} \lambda^{p-1}\left(f \lambda^{-1}\right)^{(p-\delta)(1+\varepsilon / 2)} \rho \mathrm{d} x\right)^{\frac{2-\varepsilon}{2+\varepsilon}} \\
& \times\left(\int_{B_{k, j}(\lambda) \cap\{f>\lambda / 2\}} \lambda^{-\alpha} \omega^{-2 / \varepsilon} \mathrm{d} \mu\right)^{\frac{\varepsilon(1-\varepsilon / 2)}{2+\varepsilon}}\left(\int_{B_{k, j}(\lambda)} \lambda^{-\alpha}\left(\varepsilon^{p} \omega\right)^{2 / \varepsilon} \mathrm{d} \mu\right)^{\varepsilon / 2} .
\end{aligned}
$$

Summing over all balls in the cover and using the Hölder inequality for sums, we obtain

$$
\begin{aligned}
\sum_{k=1}^{N} \sum_{j=1}^{\infty} \int_{B_{k, j}} \lambda^{p-1-2 p \varepsilon} \varepsilon^{p} \rho \mathrm{d} x & \\
\leq C\left(\int_{\{f>\lambda / 2\}} \lambda^{p-1} f^{1+\varepsilon / 2} \rho \mathrm{d} x\right)^{\frac{2-\varepsilon}{2+\varepsilon}} & \left(\int_{\{f>\lambda / 2\}} \lambda^{-\alpha} \omega^{-2 / \varepsilon} \mathrm{d} \mu\right)^{\frac{\varepsilon(1-\varepsilon / 2)}{2+\varepsilon}} \\
& \left(\int_{\{g>\lambda\}} \lambda^{-\alpha}\left(\varepsilon^{p} \omega\right)^{2 / \varepsilon} \mathrm{d} \mu\right)^{\varepsilon / 2}
\end{aligned}
$$

Here we have used the fact that on every ball $B_{k, j}(\lambda)$ the maximal function $g=M f$ is greater than $\lambda$ by definition. Next, we integrate the resulting expression with respect 
to $\lambda$ :

$$
\begin{aligned}
I_{2 \varepsilon} \leq & C\left(\int_{K}^{\infty} \mathrm{d} \lambda \int_{\{f>\lambda / 2\}} \lambda^{p-1-(p-\delta)(1+\varepsilon / 2)} f^{(p-\delta)(1+\varepsilon / 2)} \rho \mathrm{d} x\right)^{\frac{2-\varepsilon}{2+\varepsilon}} \\
& \times\left(\int_{K}^{\infty} \mathrm{d} \lambda \int_{\{f>\lambda / 2\}} \lambda^{-\alpha} \omega^{-2 / \varepsilon} \mathrm{d} \mu\right)^{\frac{\varepsilon(1-\varepsilon / 2)}{2+\varepsilon}}\left(\int_{K}^{\infty} \mathrm{d} \lambda \int_{\{g>\lambda\}} \lambda^{-\alpha}\left(\varepsilon^{p} \omega\right)^{2 / \varepsilon} \mathrm{d} \mu\right)^{\varepsilon / 2} \\
\leq & C\left(\int_{\Omega} f^{p} \rho \mathrm{d} x\right)^{\frac{2-\varepsilon}{2+\varepsilon}}\left(\int_{\Omega} K^{1-\alpha} \omega^{-2 / \varepsilon} \mathrm{d} \mu\right)^{\frac{\varepsilon(1-\varepsilon / 2)}{2+\varepsilon}}\left(\int_{\Omega} K^{1-\alpha}\left(\varepsilon^{p} \omega\right)^{2 / \varepsilon} \mathrm{d} \mu\right)^{\varepsilon / 2} \\
\leq & C\left(\int_{\Omega} f^{p} \rho \mathrm{d} x\right)^{\frac{2-\varepsilon}{2+\varepsilon}}\left(\int_{\Omega} \omega^{-2 / \varepsilon} \mathrm{d} \mu\right)^{\frac{\varepsilon(1-\varepsilon / 2)}{2+\varepsilon}}\left(\int_{\Omega}\left(\varepsilon^{p} \omega\right)^{2 / \varepsilon} \mathrm{d} \mu\right)^{\varepsilon / 2},
\end{aligned}
$$

where we have used the Hölder inequality, the Fubini theorem, and the inequalities $K>1$, $1-\alpha<0$. Using this estimate in inequality (5.12), we obtain

$$
\begin{aligned}
\int_{\Omega} \mid & \left.\nabla u\right|^{p} \rho \mathrm{d} x=\lim _{\varepsilon \rightarrow 0+} \int_{\Omega} \max (g, K)^{-2 \varepsilon}|\nabla u|^{p} \rho \mathrm{d} x \\
& \leq \frac{1}{2} \int_{\Omega}|\nabla u|^{p} \rho \mathrm{d} x+C \lim _{\varepsilon \rightarrow 0+} I_{2 \varepsilon} \\
& \leq \int_{\Omega}|\nabla u|^{p} \rho \mathrm{d} x\left[\frac{1}{2}+C \liminf _{\varepsilon \rightarrow 0+}\left(\int_{\Omega} \omega^{-2 / \varepsilon} \mathrm{d} \mu\right)^{\frac{\varepsilon(1-\varepsilon / 2)}{2+\varepsilon}}\left(\int_{\Omega}\left(\varepsilon^{p} \omega\right)^{2 / \varepsilon} \mathrm{d} \mu\right)^{\varepsilon / 2}\right] .
\end{aligned}
$$

Obviously, if the last limit is sufficiently small, then the integral on the left-hand side equals zero. Changing the variable $2 / \varepsilon=t$, we obtain the claim of the theorem under the condition that the limit (5.1) is sufficiently small.

Now we show that the limit smallness condition in (5.1) can be dropped. The boundedness of this limit is sufficient. For $\delta>0$, we define a new weight $\rho_{\delta}$ as follows:

$$
\rho_{\delta}=w_{0} w_{\delta}, \quad w_{\delta}= \begin{cases}\delta w, & w>1 / \delta \\ w, & \delta \leq w \leq 1 / \delta \\ w / \delta, & w<\delta\end{cases}
$$

The identities $H=W$ for the original weight $\rho$ and the new weight $\rho_{\delta}$ are equivalent. It is easily seen that

$$
\left\|w_{\delta} t^{-p(\cdot)}\right\|_{t} \leq t^{-\alpha} \mu(\Omega)^{1 / t}+\delta\left\|w t^{-p(\cdot)}\right\|_{t}, \quad\left\|w_{\delta}^{-1}\right\|_{t} \leq \mu(\Omega)^{1 / t}+\delta\left\|w^{-1}\right\|_{t} ;
$$

therefore, the limit (5.1) for the weight $w_{\delta}$ can be made as small as we wish by decreasing $\delta$.

The closedness property of the space $W$ in our paper is based on condition (1.2). Now we show that, under a tiny additional assumption in Theorem [6, this condition is satisfied. In the case of a constant exponent (see Theorem 3), this problem does not arise, because the weight is assumed to belong to the "global" Muckenhoupt class $A_{p}$. In essence, the additional assumption mentioned above reduces to the increased integrability of the weight $\omega_{0}^{1 /(1-p)}$ on the entire domain $\Omega$.

Lemma. Under the assumptions of Theorem 6, suppose additionally that $\omega_{0} \in A_{p(\cdot)}\left(\Omega^{\prime}\right)$, where $\Omega \Subset \Omega^{\prime}$. Then $\rho^{-1 / p} \in L^{p^{\prime}}(\Omega)$.

Proof. Since $\omega_{0}^{1 /(1-p)}$ is in $A_{\infty}\left(\Omega^{\prime}\right)$, we have $\omega_{0}^{1 /(1-p)} \in L^{1+\delta}(\Omega)$ for some $\delta>0$. Using the Young inequality $(p /(p+\delta)+\delta /(p+\delta)=1)$, we obtain

$$
\int_{\Omega}\left(\omega \omega_{0}\right)^{\frac{1}{1-p}} \mathrm{~d} x=\int_{\Omega} \omega^{\frac{1}{1-p}} \omega_{0}^{\frac{p}{1-p}} \omega_{0} \mathrm{~d} x \leq \int_{\Omega} \frac{\delta}{p+\delta} \omega^{\frac{p+\delta}{\delta(1-p)}} \omega_{0} \mathrm{~d} x+\int_{\Omega} \frac{p}{p+\delta} \omega_{0}^{\frac{1+\delta}{1-p}} \mathrm{~d} x<\infty
$$


by the assumption of the theorem.

Acknowledgments. The authors are grateful to the reviewer, whose remarks helped to improve the quality of the text.

\section{REFERENCES}

[1] V. V. Zhikov, Averaging of functionals of the calculus of variations and elasticity theory, Izv. Akad. Nauk SSSR Ser. Mat. 50 (1986), no. 4, 675-710; English transl., Math. USSR-Izv. 29 (1987), no. 1, 33-66. MR864171

[2] _ On Lavrentiev's phenomenon, Russian J. Math. Phys. 3 (1995), no. 2, 249-269. MR.1350506

[3] _ On the Lavrent' ev effect, Dokl. Akad. Nauk 345 (1995), no. 1, 10-14; English transl., Dokl. Math. 52 (1995), no. 3, 325-329. MR1374178 (97a:49020)

[4] _ On some variational problems, Russian J. Math. Phys. 5 (1997), no. 1, $105-116$. MR 1486765

[5] N. G. Meyers and J. Serrin, $H=W$, Proc. Nat. Acad. Sci. USA 51 (1964), no. 6, 1055-1056. MR 0164252

[6] V. V. Zhikov, On weighted Sobolev spaces, Mat. Sb. 189 (1998), no. 8, 27-58; English transl., Sb. Math. 189 (1998), no. 7-8, 1139-1170. MR.1669639

[7] E. M. Stein, Harmonic analysis: real-variable methods, orthogonality and oscillatory integrals, Princeton Univ. Press, Princeton, NJ, 1993. MR.1232192

[8] V. V. Zhikov, On the density of smooth functions in a weight Sobolev space, Dokl. Akad. Nauk 453 (2013), no. 3, 247-251; English transl., Dokl. Math. 88 (2013), no. 3, 669-673. MR3185296

[9] M. D. Surnachev, On the density of smooth functions in a weighted Sobolev space with a variable exponent, Dokl. Akad. Nauk 455 (2014), no. 1, 18-22; English transl., Dokl. Math. 89 (2014), no. 2, 146-150. MR3237615

[10] O. Kováčik and J. Rákosník, On spaces $L^{p(x)}(\Omega)$ and $W^{k, p(x)}(\Omega)$, Czechoslovak Math. J. 41 (1991), no. 6, 592-618. MR1134951 (92m:46047)

[11] X. O. Fan, Regularity of nonstandard Lagrangians $f(x, \xi)$, Nonlinear Anal. 27 (1996), no. 6, 669678. MR 1399067

[12] X. Fan and D. Zhao, On the spaces $L^{p(x)}(\Omega)$ and $W^{m, p(x)}(\Omega)$, J. Math. Anal. Appl. 263 (2001), no. 2, 424-446. MR1866056

[13] S. G. Samko, Density of $C_{0}^{\infty}\left(\mathbb{R}^{n}\right)$ in the generalized Sobolev spaces $W^{m, p(x)}\left(\mathbb{R}^{n}\right)$, Dokl. Akad. Nauk 369 (1999), no. 4, 451-454; English transl., Dokl. Math. 60 (1999), no. 3, 382-385. MR.1748959

[14] L. Diening, Riesz potentials and Sobolev embeddings on generalized Lebesgue and Sobolev spaces $L^{p(\cdot)}$ and $W^{k, p(\cdot)}$, Math. Nachr. 268 (2004), 31-43. MR2054530

[15] _ Maximal functions in generalized $L^{p(\cdot)}$ spaces, Math. Inequal. Appl. 7 (2004), no. 2, 245-254. MR2057643(2005k:42048)

[16] _ Maximal function on Musieliak-Orlicz spaces and generalized Lebesgue spaces, Bull. Sci. Math. 129 (2005), no. 8, 657-700. MR2166733

[17] D. Cruz-Uribe, A. Fiorenza, and C. J. Neugebauer, The maximal function on variable $L^{p}$ spaces, Ann. Acad. Sci. Fen. Math. 28 (2003), no. 1, 223-238, and corrections in 29 (2004), 247-249. MR.1976842 (2004c:42039)

[18] D. Cruz-Uribe, L. Diening, and A. Fiorenza, A new proof of the boundedness of maximal operators on variable Lebesgue spaces, Boll. Unione Mat. Ital. (9) 2 (2009), no. 1, 151-173. MR2493649

[19] L. Pick and M. Růžička, An example of a space $L^{p(x)}$ on which the Hardy-Littlewood maximal operator is not bounded, Expo. Math. 19 (2001), no. 4, 369-371. MR1876258

[20] D. Cruz-Uribe, A. Fiorenza, J. M. Martell, and C. Pèrez, The boundedness of classical operators on variable $L^{p}$ spaces, Ann. Acad. Sci. Fenn. Math. 31 (2006), no. 1, 239-264. MR2210118

[21] V. V. Zhikov, On the density of smooth functions in Sobolev-Orlicz spaces, Zap. Nauchn. Sem. S.-Peterburg. Otdel. Mat. Inst. Steklov. (POMI) 310 (2004), 67-81; English transl., J. Math. Sci. (N.Y.) 132 (2006), no. 3, 285-294. MR2120185

[22] L. Diening, P. Harjulehto, P. Hästö, and M. Růžička, Lebesgue and Sobolev spaces with variable exponents, Lecture Notes in Math., vol. 2017, Springer, Heidelberg, 2011. MR2790542

[23] X. Fan, J. Shen, and D. Zhao, Sobolev embedding theorems for spaces $W^{k, p(x)(\Omega), ~ J . ~ M a t h . ~ A n a l . ~}$ Appl. 262 (2001), no. 1, 749-760. MR1859337

[24] V. V. Zhikov, On variational problems and nonlinear elliptic equations with nonstandard growth conditions, Probl. Mat. Anal. 54 (2011), 23-112; English transl., J. Math. Sci. 173 (2011), no. 5, 463-570. MR2839881 
[25] Yu. A. Alkhutov and V. V. Zhikov, The Hölder property of solutions of an elliptic equation, Sovrem. Mat. Prilozen. 10 (2003), 8-21; English transl., J. Math. Sci. (N.Y.) 129 (2005), no. 1, 3523-3536. MR:2162683

[26] P. Harjulehto, P. Hästö, M. Koskenoja, and S. Varonen, Sobolev capacity on the space $W^{1, p(\cdot)}\left(\mathbb{R}^{n}\right)$, J. Funct. Spaces Appl. 1 (2003), no. 1, 17-33. MR2011498

[27] L. Diening and P. Hästö, Muckenhoupt weights in variable exponent spaces; http://www.helsinki.fi/〜hasto/pp/p75_submit.pdf.

[28] D. Cruz-Uribe, L. Diening, and P. Hästö, The maximal operator on weighted variable Lebesgue spaces, Fract. Calc. Appl. Anal. 14 (2011), no. 3, 361-374. MR2837636

[29] D. Cruz-Uribe and A. Fiorenza, Variable Lebesgue spaces. Foundations and harmonic analysis, Birkhäuser, Heidelberg, 2013. MR3026953

Vladimir State University, Stroitelei Ave., 600000 Vladimir, Russia

E-mail address: zhikov@vlsu.ru

Aeroacustics Laboratory, Keldysh Institute of Applied Mathematics, Russian Academy of Sciences, 4 Miusskaya square, 125047 Moscow, Russia

E-mail address: peitsche@yandex.ru

Received 15/SEP/2014

Translated by N. RASTEGAEV 